\title{
Photonic crystal waveguides based on an antiresonant reflecting platform
}

\author{
Lavrinenko, Andrei; Frandsen, Lars Hagedorn; Fage-Pedersen, Jacob; Borel, Peter Ingo
}

Published in:

Proceedings of 2005 7th International Conference on Transparent Optical Networks

Link to article, DOI:

10.1109/ICTON.2005.1505802

Publication date:

2005

Document Version

Publisher's PDF, also known as Version of record

Link back to DTU Orbit

Citation (APA):

Lavrinenko, A., Frandsen, L. H., Fage-Pedersen, J., \& Borel, P. I. (2005). Photonic crystal waveguides based on an antiresonant reflecting platform. In Proceedings of 2005 7th International Conference on Transparent Optical Networks (Vol. 1, pp. 273-276). IEEE. https://doi.org/10.1109/ICTON.2005.1505802

\section{General rights}

Copyright and moral rights for the publications made accessible in the public portal are retained by the authors and/or other copyright owners and it is a condition of accessing publications that users recognise and abide by the legal requirements associated with these rights.

- Users may download and print one copy of any publication from the public portal for the purpose of private study or research.

- You may not further distribute the material or use it for any profit-making activity or commercial gain

- You may freely distribute the URL identifying the publication in the public portal 


\title{
Photonic Crystal Waveguides Based on an Antiresonant Reflecting Platform
}

\author{
Andrei V. Lavrinenko, Lars H. Frandsen, Jacob Fage-Pedersen, Peter I. Borel \\ Research Center COM, NanoDTU, Technical University of Denmark, 345v, DK-2800, Kgs. Lyngby, Denmark \\ Tel: (45) 4525 6392, Fax: (45) 4593 6581, e-mail: ala@com.dtu.dk
}

\begin{abstract}
We apply the antiresonant reflecting layers arrangement to silicon-on-insulator based photonic crystal waveguides. Several layered structures with different combinations of materials $\left(\mathrm{Si}-\mathrm{SiO}_{2}, \mathrm{Si}_{3} \mathrm{~N}_{4}-\mathrm{SiO}_{2}\right)$ and layer topology have been analysed. Numerical modelling using the 3D Finite-Difference Time-Domain method reveals promising low-loss results with potential for competing with membrane-like photonic crystal waveguides.
\end{abstract}

Keywords: photonic crystal waveguides, Photonic Band Gap (PBG), Antiresonant Reflecting Optical Waveguides (ARROW), Finite-Difference Time-Domain (FDTD) method.

\section{INTRODUCTION}

The trend of miniaturising optical integrated contours has been boosted by the concept of photonic band gap (PBG) materials, in which cavities and waveguides with novel properties can be created by introducing pointlike or line defect states in the photonic crystal lattice. However, there are certain obstacles on the way towards the integrated circuit based on PBG structures. Specifically, propagation losses in photonic crystal waveguides (PCWs) are too high in comparison to rib or ridge waveguides. Thus, the reduction of losses in straight PCWs is a key topic on the way of designing integrated photonic circuits based thoroughly on PBG elements.

Several designs addressing the high propagation losses of PCWs have been suggested. The most successful one is the membrane-like concept utilising the high index ratio of a PCW host material (e.g. Si, GaAs or InP) and air above and below the channel [1-3]. Presently, the membrane-like waveguides give the lowest losses for PCWs, however, they have their drawbacks in mechanical stiffness and problems in the vertical arrangement of the elements. Another design approach, based on the silicon-on-insulator (SOI) platform [3], utilises a so-called W0.7 waveguide where the width of the waveguide core, arising from removing one row of holes in the crystal lattice, is squeezed to $70 \%$ of the original width. The narrow waveguide core causes design problems when introducing bends, coupling, and splitting regions, hence, the W0.7 PCW is not an attractive solution for complex circuits. The same problem exists for the asymmetric type B PCW [4] formed by stacking two 2D photonic crystal structures with triangular lattices in an out-of-phase arrangement, leaving a low-loss W1 PCW in between them. This layout has not yet been characterised in any experiments on the SOI platform and may still possess potential for utilising the dispersion properties of straight PCW. Ultra low-loss guiding in biperiodic PCWs was reported in Ref. [5], where the rows of holes adjacent to the W1 PCW are changed in size and placed with a different period than the bulk holes of the lattice. However, such bi-periodic PCWs have dispersion properties similar to ordinary ridge waveguides, and may, therefore, loose their uniqueness. Finally, waveguides in $3 \mathrm{D}$ photonic crystal structures can be mentioned, which by definition have intriguing possibilities to guide light with extremely low losses, see e.g. numerical results in Ref. [6]. However, 3D lattices are extremely difficult to fabricate and lie beyond our scope of planar technology applications.

The main source of propagation losses in 2D PCWs has been believed to be the guided modes' position above the light line, since modes above the light line easily can couple out of the plane to the continuum of radiation modes of the upper or lower cladding material. The membrane-like PCWs have the biggest possible parts of the dispersion curves for the guided modes below the light line since the relevant light line is that of air. Hence, they show a low loss in a broad wavelength range. For PCWs based on the SOI platform the silica line leaves less of the dispersion curves below the radiation continuum and, thus, gives rise to larger propagation losses. Here, we propose an alternative, namely to employ the so-called antiresonant reflecting optical waveguides (ARROW) concept to SOI-based PCWs to reduce the out-of-plane scattering losses.

\section{APPLICATION OF THE ARROW CONCEPT IN PHOTONIC CRYSTAL WAVEGUIDES}

The antiresonant reflecting concept stems from the work by Guguay et al [7] (Fig. 1a). The thickness of the first silicon cladding beneath the silica core is deliberately chosen to provide high reflectance by the interference principle, like a Fabry-Perot resonator, which have broad reflecting windows on antiresonant wavelengths and sharp transmission peaks on resonant wavelengths. In order to enhance the resonance effect the silicon cladding is underpinned by a second cladding layer made from silica. The condition for resonant reflection of the evanescent fields penetrating through the first cladding is fulfilled by making the thickness of the silicon cladding approximately half that of the silica core. Thus, in total, a reflection around $99.96 \%$ at the bottom

The work reported in the paper was supported by the PIPE project of the Danish Technical Research Council. 
interface of the core arises [7]. Under the zero-order approximation, the claddings are nothing else but $\lambda / 4$ plates with respect to the transverse component of the wavevector [8]. An attempt to apply the ARROW concept with PCWs has recently been made [9], but the authors chose the core thickness to be 7 microns to improve the coupling between PCWs and fibres. However, etching 7 microns deep holes with smooth sidewalls is a very challenging problem.

The original ARROW idea was further developed in [10-12] where new generations of ARROW structures with a high index core and a low index first cladding were proposed and characterised. These are the so-called ARROW-B (Fig.1, b) and ARROW-C configurations, which are applicable for SOI-based PCWs.

One significant issue has to be stressed concerning ARROW-B based PCWs. A standard core thickness of classical ARROW structures is about 4-5 microns or more [10-12], and losses rapidly decrease with increasing core thickness. On the contrary, the thickness of the silicon core in an SOI-based PCW is severely limited by the requirement of a reasonable size of the photonic band gap. The gap-midgap frequency ratio was analysed in paper [13] and it was found that the optimal slab thickness for photonic crystal structures defined by a triangular lattice of holes is $0.7 a$, where $a$ is the lattice constant. If the slab thickness is increased the gap-midgap frequency ratio is decreased. Keeping in mind that promising applications of PCWs such as submicron-radii bends, splitters, and couplers are totally dependent on the PBG effect, we have to restrict ourselves to the case of the $\sim 0.7 a$ thick silicon core, which inevitably triggers the propagation loss to increase. The main purpose of our investigation is to verify the usefulness of the ARROW concept in order to decrease the propagation losses in SOI-based PCWs.

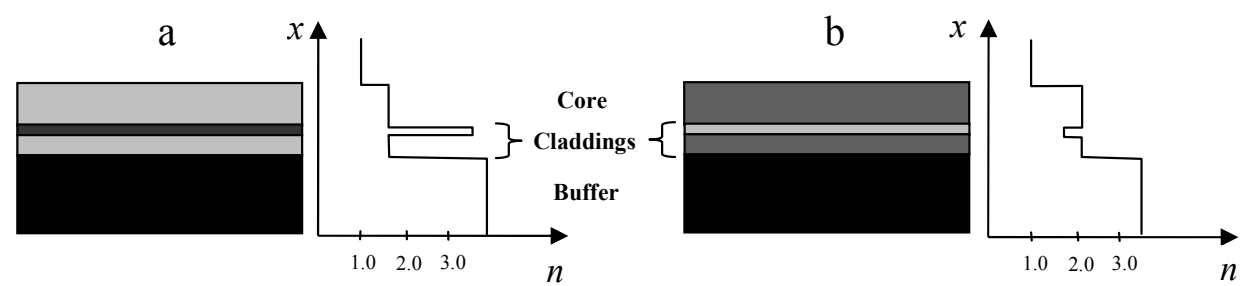

Figure 1. Structures and examples of index profile: a) conventional ARROW, b) ARROW-B.

\section{CHARACTERISATION OF ARROW-B PHOTONIC CRYSTAL WAVEGUIDES}

There is a standard procedure for direct calculation of propagation losses using numerical techniques [6, 14]. The transmission is evaluated for waveguides of different lengths and the damping constant $\alpha$ is obtained as

$$
\alpha=\ln \left(\frac{T_{2}}{T_{1}}\right) \frac{1}{L_{1}-L_{2}},
$$

where $T_{1}$ and $T_{2}$ are the transmission levels through waveguides of length $L_{1}$ and $L_{2}$, respectively.

In cases where propagation losses are very low, it is impossible to extract proper values due to fringes in the transmission spectra calculated using the FDTD technique. The transmission through longer waveguides can appear to be larger than the transmission through shorter waveguides, and this produces artificial negative losses $[6,14]$. The only well-established remedy is to increase the propagating distance by an order of magnitude. Unfortunately, photonic crystal systems with about $1 \mathrm{~mm}$ length require special hardware to allocate field arrays during FDTD modelling. So typically, systems simulated by standard computers are no more than $100 \mu \mathrm{m}$ in length. For this reason we choose here to show transmission spectra only, while the refinement of the damping constant requires more calculation efforts.

\subsection{Standard ARROW-B PCW design with a thin Si core}

First we investigated the structure shown in Fig.1b with the following parameters: The core is made of Si having index of refraction $n_{c}=3.5$. The lattice constant and hole diameter were chosen to position the central PBG wavelengths near $1.33 \mu \mathrm{m}: a=0.336 \mu \mathrm{m}, D=0.185 \mu \mathrm{m}$. The FDTD resolution was 16 points per lattice constant. The core thickness was taken to be approximately twice the optimal value given in [13] $-0.5 \mu \mathrm{m}$. The indices of refraction of the first and the second claddings (glass and Si) are $n_{1}=1.46$ and $n_{2}=3.5$, respectively. The modelled system is surrounded by a thin layer of air above the core and a thin glass buffer layer below the second cladding.

The first cladding thickness may be chosen in a broad range due to a monotonic reduction of losses with increase of this thickness [12]. Unfortunately there is a practical limit to its size because of RAM memory requirements for testing long enough waveguides, and we took $d_{l}=0.7 \mu \mathrm{m}$ as a default value.

The second cladding thickness was calculated accordingly to the formula derived by Baba et al [12] 


$$
d_{2}=\frac{d_{c e}}{2}(2 N+1), N=0,1,2, \ldots,
$$

where the effective core thickness $d_{c e}$ was calculated from

$$
d_{c e}=d_{c}+\frac{\lambda}{2 \pi \sqrt{n_{c}^{2}-n_{0}^{2}}}+\frac{\lambda}{2 \pi \sqrt{n_{c}^{2}-n_{1}^{2}} \tanh \left(\frac{2 \pi}{\lambda} d_{1} \sqrt{n_{c}^{2}-n_{1}^{2}}\right)}
$$

In this way we obtained $d_{2}=0.315 \mu \mathrm{m}$. Transmission spectra for the PCWs with different lengths from $20 a$ to $135 a$ are plotted in Fig. 2.

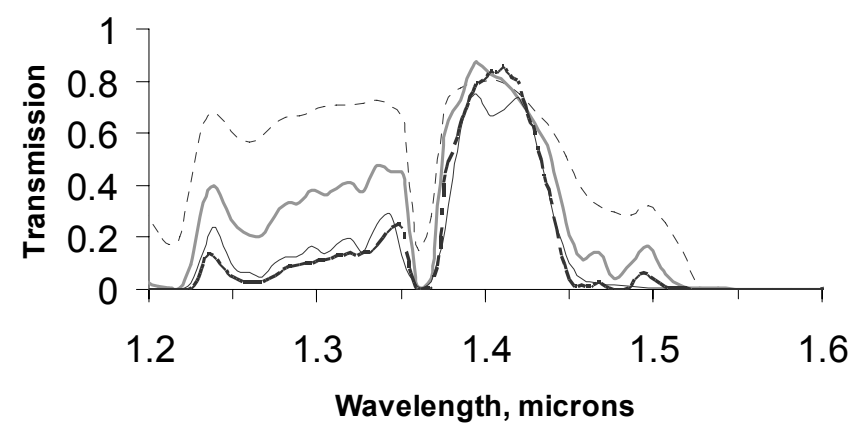

Figure 2. Transmission spectra for the ARROW-B PCWs of different lengths: 20 a (thin dashed line), 60a (thick grey line), 100a (thin solid line), and 135a (solid-dashed thick black line).

The main transmission window spans from $1.23 \mu \mathrm{m}$ to $1.45 \mu \mathrm{m}$. It decreases rapidly with increasing propagation distance everywhere except at the resonant range around $1.4 \mu \mathrm{m}$. It is easy to assess the losses in the region $1.23-1.36 \mu \mathrm{m}$, where the scattering occurs primarily due to the location of modes above the silica light line; the losses here are higher than $100 \mathrm{~dB} / \mathrm{mm}$. On the other hand, the attenuation of the transmission near the resonant wavelength is so small that it cannot be evaluated; the transmitted intensity simply fluctuates around a mean value of 0.8 . In this region the longest waveguide almost everywhere has higher transmission than the shortest one. Even averaging data over the transparent window does not provide clear positive numbers.

\subsection{Standard ARROW-B PCW design with a thin $\mathrm{Si}_{3} \mathrm{~N}_{4}$ core}

The three-layer $\mathrm{Si}_{-} \mathrm{SiO}_{2}$ design placed on top of a $\mathrm{SiO}_{2}$ buffer promises low loss, but poses fabrication challenges. Thus we may look for alternative material compositions, like $\mathrm{Si}_{3} \mathrm{~N}_{4}$ glass on a Si buffer. So we have modelled a structure consisting of a $0.280 \mu \mathrm{m}$ thick air layer, a $0.504 \mu \mathrm{m}$ thick core $\left(n_{c}=2.0\right)$, a $0.672 \mu \mathrm{m}$ thick first cladding $\left(n_{1}=1.46\right)$, a $0.392 \mu \mathrm{m}$ thick second cladding $\left(n_{2}=2.0\right)$ and a $0.140 \mu \mathrm{m}$ thick Si buffer. The parameters of the lattice are: $a=0.448 \mu \mathrm{m}$ and $D=0.25 \mu \mathrm{m}$, the FDTD resolution is 16 points per lattice constant. All thicknesses and the lattice constant were rounded to the nearest integer multiple factor of FDTD grid resolution, which is $28 \mathrm{~nm}$ in this case. Transmission spectra for PCWs of lengths $20 a, 100 a$, and $170 a$ are presented in Fig. 3.

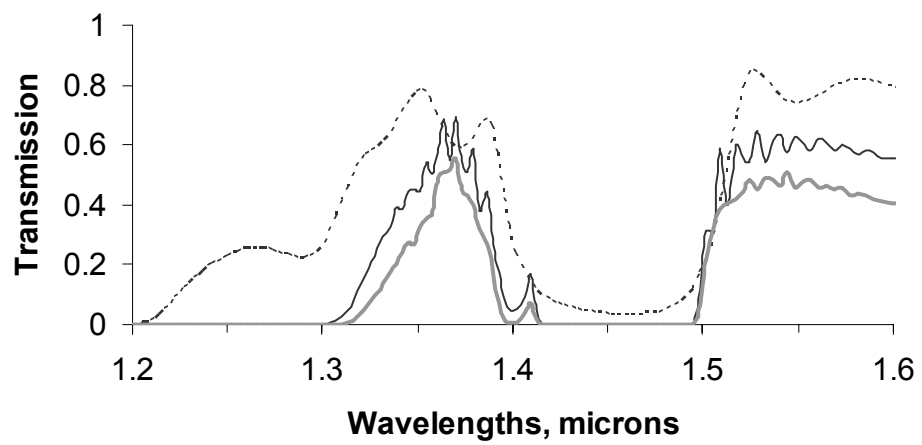

Figure 3. Transmission spectrum for the ARROW-B PCW with $\mathrm{Si}_{3} \mathrm{~N}_{4}$ core. The lengths of the waveguides are 20 a (thin dashed line), 100a (thin solid line), and 170a (thick grey line).

It is clear that the PBG range is narrower in the case of lower index dielectric, compared to the $\mathrm{Si}_{-} \mathrm{SiO}_{2}$ structure. The average transmission is also lower. At the resonant peak near $1.37 \mu \mathrm{m}$ the damping coefficient can be evaluated to approximately $12 \mathrm{~dB} / \mathrm{mm}$. This value is quite high, but it can certainly be improved by optimising the thicknesses of the first and the second cladding layers. Such an optimisation, requiring thicker layers, is expected to be technologically feasible. 


\subsection{Simplified ARROW-B PCW design}

To meet fabrication criteria of the SOI platform we modelled an ARROW-B design with one significant simplification - the lack of the second cladding. The remaining (low-index) cladding was placed on the high index buffer. The sandwich for modelling now consists of the air layer, $0.504 \mu \mathrm{m}$ thick Si core $\left(n_{c}=3.5\right)$, $0.756 \mu \mathrm{m}$ thick first cladding $\left(n_{1}=1.46\right)$, and $0.504 \mu \mathrm{m}$ thick Si buffer. The parameters of the lattice are $a=0.420 \mu \mathrm{m}, D=0.32 \mu \mathrm{m}$, and FDTD resolution 15 points per lattice constant. All thicknesses and the lattice constant were rounded to the nearest integer multiple factor of FDTD grid resolution $(28 \mathrm{~nm})$. Transmission spectra for $50 \mu \mathrm{m}$ and $100 \mu \mathrm{m}$ long PCWs are presented in Fig. 4. In the proximity of the resonance point at $1.54 \mu \mathrm{m}$ losses are estimated to lie in the interval $0 \pm 4 \mathrm{~B} / \mathrm{mm}$. For comparison we also supply the transmission spectrum for an ordinary W1 PCW based on the ordinary SOI platform. It is $3-4 \%$ lower in transmission, but more investigations are needed here to understand exactly the resonant behaviour of the ARROW-B type design.

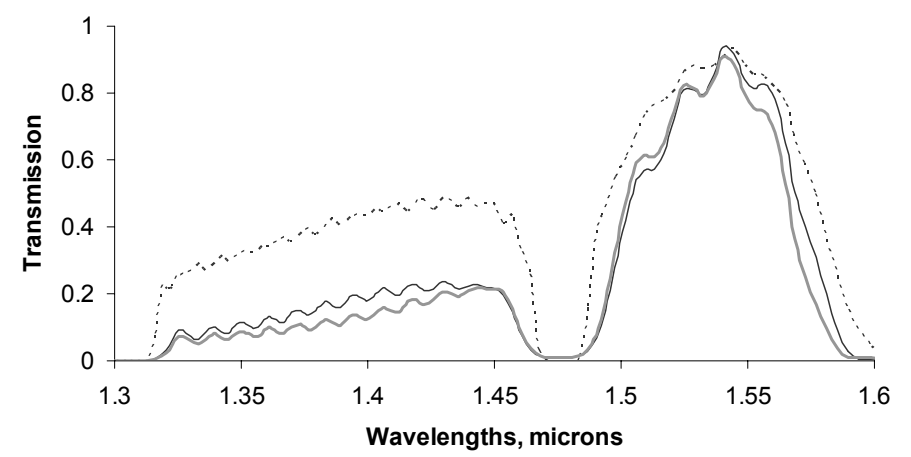

Figure 4. Transmission spectra for the simplified ARROW-B PCWs with Si cores having lengths of $50 \mu$ m (thin

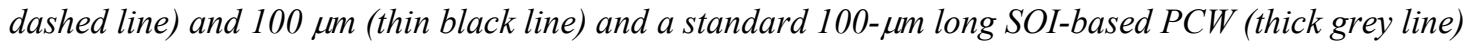

\section{CONCLUSIONS}

We have addressed an antiresonant reflection waveguiding platform to be employed within PCW designs. A strong resonance dependence of the transmission spectrum is clearly seen. Structures with a lowered core index have larger losses. We checked also a simplified design without the second cladding with high index buffer. All results signal clearly that the ARROW-B concept has large potential for use within photonic crystals circuits. More thorough investigations are needed, especially with waveguides of larger lengths.

\section{REFERENCES}

[1] S.J. McNab, et al.: Ultra-low loss photonic integrated circuit with membrane-type photonic crystal waveguides, Opt. Express, vol.11 pp. 2927-2939, 2003.

[2] Y.Sugimoto, et al.: Low propagation loss of $0.76 \mathrm{~dB} / \mathrm{mm}$ in GaAs-based single-line-defect two-dimensional photonic crystal slab waveguides up to $1 \mathrm{~cm}$ in length, Opt. Express, vol.12 pp.1090-1097, 2004.

[3] M. Notomi, et al.: Waveguides, resonators and their coupled elements in photonic crystal slabs, Opt. Express, vol.12 pp.1551-1561, 2004.

[4] N. Susa: The large bandwidth and large group velocity for the single guided mode in an asymmetric photonic crystal slab waveguide, Japanese J. Appl. Phys., vol.42B pp. L724-L727, 2003.

[5] A. Jafarpour, et al.: Large-bandwidth ultra-low-loss guiding in bi-periodic photonic crystal waveguides, Appl. Phys. B, vol.79 pp.409-414, 2004.

[6] Y. Watanabe, N. Yamamoto, K. Komori: Numerical analysis of waveguides in three-dimensional photonic crystal with finite thickness, Japanese J. Appl. Phys., vol. 43 pp. 2015-2018, 2004.

[7] M.A.Duguay, et al.: Antiresonant reflecting optical waveguides in $\mathrm{SiO}_{2}-\mathrm{Si}$ multilayer structures, Appl. Phys. Lett., vol. 49 pp. 13-15, 1986.

[8] M.H. Sheng, H.W. Chang: Accurate first-order leaky-wave analysis of antiresonant reflecting optical waveguides, Appl. Opt., vol.44 pp. 751-764, 2005.

[9] Y.-L. Yang, et al.: ARROW-based photonic crystal waveguides for efficient coupling with fibers, in Proc. of ECIO2005, Grenoble, France, April 2005, pp. 642-645, 2005.

[10] T. Baba, Y. Kokubun: New polarization-insensitive antiresonant reflecting optical waveguide (ARROW-B), IEEE Photonics Technol. Lett., vol.1 pp. 232- 234, 1989.

[11] R.A. Soref, et al.: Silicon antiresonant reflecting optical waveguides, Opt. Lett., vol.15 pp. 792-794, 1990.

[12] T. Baba, Y. Kokubun: Dispersion and radiation loss characteristics of antiresonant reflecting optical waveguides numerical results and analytical expressions, IEEE J. Quantum Electron., vol.281 pp. 1689- 1700, 1992.

[13] S.G. Johnson, et al:: Guided modes in photonic crystal slabs, Phys. Rev. B, vol.60 pp. 5751-5758, 1999.

[14] M.J. Cryan, et al.: Calculation of losses in 2-D photonic crystal membrane waveguides using the 3-D FDTD method, IEEE Photonics Technol. Lett., vol.17 pp. 58-60, 2005. 